\section{1-5.5 DETERMINANTS OF HIGH FOLATE CONCENTRATION IN THE CANADIAN POPULATION}

doi:10.1136/jech.2011.142976a.40

\begin{abstract}
${ }^{1,2} \mathrm{C}$ K Colapinto, ${ }^{*}{ }^{3,4} \mathrm{D} \mathrm{L} \mathrm{O}$ 'Connor, ${ }^{2} \mathrm{~L}$ Dubois, ${ }^{1,5} \mathrm{M}$ S Tremblay. ${ }^{1}$ Children's Hospital of Eastern Ontario Research Institute, Healthy Active Living and Obesity Research Group, Ottawa, Ontario, Canada; ' 2 University of Ottawa Institute of Population Health, Ottawa, Ontario, Canada; ${ }^{3}$ The Hospital for Sick Children, Toronto, Ontario, Canada; ${ }^{4}$ University of Toronto, Department of Nutritional Sciences, Faculty of Medicine, Toronto, Ontario, Canada; ${ }^{5}$ University of Ottawa, Department of Pediatrics, Faculty of Medicine, Ottawa, Ontario, Canada
\end{abstract}

Introduction Canadians' red blood cell (RBC) folate has shifted towards high concentrations ( $>1360 \mathrm{nmol} / 1$ based on the 97th percentile of Americans post-fortification (NHANES)). Determinants of these high concentrations are poorly understood, though an association has been posited between high intakes of folic acid and adverse health outcomes. This research investigated determinants of high folate concentrations in Canadians.

Methods RBC folate concentrations from the nationally representative Canadian Health Measures Survey were examined in participants aged $6-79$ years $(\mathrm{N}=5248)$. The population was described using frequencies and percentages. Sociodemographic, behavioural and clinical determinants of high RBC folate concentrations were examined using univariate and separate multiple logistic regression models controlling for age and household income. Results The greatest proportion of high concentrations occurred in females (42.5\%), higher age groups (6-11 years (36.4\%), $12-19$ years $(25.6 \%), 20-39$ years (32.9\%), 40-59 years $(44.5 \%)$, $60-79$ years $(53.6 \%)$ ) and higher income quartiles (33.5\% (Q1), $37.6 \%$ (Q2), 41.6\% (Q3), 46.6\% (Q4)). Folic acid containing supplement users had a greater prevalence of high concentrations $(62.8 \%)$ than non-users (37.2\%). Prevalence of high concentrations climbed with increasing intake of fruit/vegetables ( $>3$ times per day $(46.8 \%))$ and grain products ( $>3$ times per day $(45.5 \%))$. Never smokers $(39.5 \%)$ and former smokers $(49.1 \%)$ had a greater prevalence of high concentrations than daily smokers (28.4\%). Detailed regression results will be presented at the conference.

Conclusion Determinants of high folate concentrations should be considered when refining folic acid supplementation and fortification policies. Future research on the relationship between high folate concentrations and health outcomes is warranted.

\section{1-5.6 FORECASTING DIABETES PREVALENCE: VALIDATION OF A SIMPLE MODEL WITH FEW DATA REQUIREMENTS}

doi:10.1136/jech.2011.142976a.41

${ }^{1} \mathrm{M}$ O'Flaherty, ${ }^{2} \mathrm{~J}$ Critchley, ${ }^{3} \mathrm{~S}$ Wild, ${ }^{2} \mathrm{~N}$ Unwin, ${ }^{1} \mathrm{~S}$ Capewell. ${ }^{1}$ MEDCHAMPS Collaboration, Liverpool, UK; ${ }^{2}$ MEDCHAMPS Collaboration, Newcastle, UK; ${ }^{3}$ University of Edinburgh, Edinburgh, UK

Introduction Current projections of diabetes prevalence are mostly based on demographic change. Explicitly including trends in obesity and other risk factors could improve the accuracy of the projections and assist in evaluating policy options for prevention.

Methods The model integrates population, obesity and smoking trends to estimate future diabetes prevalence using a Markov approach. Model parameters were derived from the literature, except for diabetes incidence which was estimated using DISMOD from the baseline estimation of diabetes prevalence. We developed a model for the US population (2000-2006), and validated the model outputs (NHANES prevalence and projections using a different model).

Results US Diabetes mellitus prevalence estimated by the model (aged $25+$ ) was $9.7 \%$ in $2000-2002(7.8 \%-11.6 \%)$, increasing to $10.7 \%(8.6 .3 \%-12.7 \%)$ by $2003-2006$. Comparisons of the model results with the observed prevalence in the NHANES survey showed a close fit to the observed estimates (NHANES prevance 2003-2006
$10.3 \%, 9.3 \%-11.3)$. The forecasts for 2030 was 19 19.3\% (15.3\%$23.0 \%$ ). A different model (Narayan et al) for the same period and age group were $20.2 \%, 18.8 \%-21.6 \%$. We tested the model for the England and Wales population obtaining a similar performance.

Conclusions This model provides a reasonably close estimate of diabetes prevalence for the USA over the 2000-2006 period, compared with contemporary independent prevalence surveys in the same population and with a different model. Because of its few data requirements, the approach is now being tested in different middle income countries as a potential global diabetes prevalence forecast tool.

\subsection{COHORT STUDIES AROUND THE WORLD: METHODOLOGIES, RESEARCH OUESTIONS AND INTEGRATION TO ADDRESS THE EMERGING GLOBAL EPIDEMIC OF CHRONIC DISEASES}

\section{Chair: Prof Donna Spiegelman, USA Discussant: Prof. David Hunter, USA $01-6.1$
THE SHANGHAI WOMEN'S AND MEN'S HEALTH
STUDS}

doi:10.1136/jech.2011.142976a.42

Xiao-ou Shu.* Vanderbilt University, Nashville, Tennessee, USA

Rapid economic developments accompanied by environmental and lifestyle changes over the last 3 decades in China have resulted in dramatic increases in the incidence of chronic diseases such as cancer and cardiovascular disease. As a result, cancer and cardiovascular disease are two of the leading causes of death in China. This change in disease spectrum presents an enormous challenge to public health practitioners and policy makers in designing cost-efficient strategies for disease prevention. To identify reasons for the increased risk of chronic disease in China and investigate etiologic hypotheses that cannot be adequately evaluated in other populations, we launched the Shanghai Women's Health Study in 1996 and the Shanghai Men's Health Study in 2001. In collaboration with community health workers, the Shanghai Women's Health Study recruited 75049 women aged 40-70 from 7 communities in urban Shanghai between 1997 and 2000 with an overall response rate of $92 \%$. Using a similar protocol, the Shanghai Men's Health Study recruited 61500 men aged 40-74 from 8 communities in Shanghai with a response rate of $75 \%$ for study participation. Biological samples were collected from the vast majority of study participants. All study participants were interviewed using a structured questionnaire to obtain information related to their usual dietary intake, physical activity, and other lifestyle factors. These two cohorts are being followed through a combination of biennial in-person surveys and record linkage with the Shanghai Cancer Registry and Shanghai Vital Statistics database. In addition to ascertaining health outcomes, we also obtain exposure data as part of the follow-up surveys.

\section{$01-6.2$ \\ THE ESMAESTRAS STUDY: A LARGE COHORT STUDY AMONG MEXICAN TEACHERS}

doi:10.1136/Jech.2011.142976a.43

I Romieu.* International Agency for Research on Cancer, Lyon, France \& Mexico City, Mexico

The EsMaestras study is a prospective cohort study which enrolled female teachers 25 years and older from 12 states of Mexico including urban and rural schools. The main objective of the cohort is to evaluate life styles and environmental risk factors related to chronic diseases with major focus on breast and reproductive 
cancers, diabetes and cardiovascular disease in a society with rapidly changing life styles, increasing sedentarity and westernized diet. The main exposures of interest are dietary intake, physical activity, obesity and environmental exposure in particular endocrine disruptor chemicals as well as gene by environment interaction. The study started in 2006 enrolling teachers from 2 states (Veracruz and Jalisco) in 2008-2009, 10 others states were included. To date the study enrolled close to 116000 women. Overall the response rate has been $64 \%$, and follow-up at 4 years $71 \%$. Information on sociodemographic, lifestyle, reproductive history, medical history, anthropometry, physical activity and diet is collected through biennial questionnaire. In addition in a subsample of the study population detailed information including mammographic density, bone density, blood pressure, spirometry, impedance and anthropometric measurements, blood and urinary samples as well as more detailed questionnaire on lifestyle. This large cohort with a high variability in lifestyle and fast changing habits will provide very valuable information on risk factors of chronic diseases in female.

\section{1-6.3 LIFE STYLE FACTORS \& BODY MASS INDEX AS PREDICTOR OF MORTALITY: FINDINGS FROM A COHORT STUDY IN INDIA}

doi:10.1136/jech.2011.142976a.44

\section{K Ramadas.* Regional Cancer Centre, Trivandrum, India}

A population based cohort study including 167343 adults aged 34 and above living in a rural area were recruited and followed-up during January 1996-December 2004 as part of an ongoing oral cancer screening programme. Blood pressure and body mass index (BMI) were measured at baseline and at 3 year intervals. Demographic details and lifestyle factors were also collected through a questionnaire. These subjects were actively followed-up for vital status by field workers through municipal death registers, records collected from social and religious organisations, and during repeated house visits and cause of deaths were determined by verbal autopsy.

The mortality risks associated with chewing (and 95\% CIs), after adjusting for age, sex, socio-economic dietary variables, and for other habits, were 0.90 (0.86 to 0.94$)$ for all-cause, 1.07 (0.94 to 1.22$)$ for cancer and 1.22 (1.04 to 1.44$)$ for tobacco-related cancer; with smoking the respective mortality risks were 1.31 (1.24 to 1.39 ), 1.63 (1.37 to 1.94$)$ and 1.68 (1.36 to 2.08); and with alcohol use the risks were 1.13 (1.06 to 1.20), 1.32 (1.11 to 1.57 ) and 1.47 (1.19 to 1.80 ). Reduced risk of all-cause mortality by chewing was observed only in the 60-84 years old group $(0.90$ (0.85 to 0.94)), and detrimental effects of chewing on cancer mortality were shown in the young and middle-age groups: $34-39$ years old (1.33 (0.67 to 2.65)), and $40-59$ years old (1.26 (1.03 to 1.55$)$ ). An analysis of the association between BMI and change in weight in relation to mortality risk showed that low BMI was found to be a predictor of mortality, while high BMI was not.

\section{1-6.4 PARTNERSHIP FOR COHORT RESEARCH AND TRAINING (PaCT): PILOT STUDY, SOUTH AFRICA}

doi:10.1136/jech.2011.142976a.45

J Volmink.* Stellenbosch University, Stellenbosch, South Africa

An epidemic of chronic, non-communicable diseases is growing in Africa. By 2030, according to the WHO, three of the top four causes of death in low-income countries will be heart disease, stroke, and chronic lung disease. No large scale epidemiologic studies investigating chronic diseases have been conducted in Africa.

PaCT, a component of the Global Epidemiology Initiative established at the Harvard School of Public Health, is an ambitious project which will investigate chronic, non-communicable diseases among 500000 people from four African countries over the next 20 years. This initiative will also provide training opportunities for African researchers. PaCT includes scientists from universities in South Africa, Uganda, Tanzania, Nigeria, as well as Harvard University. Pilot studies are being conducted in all four of these countries.

An initial cross-sectional baseline survey, with 6 month longitudinal follow-up was planned. We aimed to recruit 800-1000 school teachers employed at public schools within the CapeTown metropolitan area. After returning a self-administered questionnaire and completed consent forms, teachers were visited at the schools by trained nurses who took physical measurements (weight, height, waist circumference and blood pressure) and collected biological (blood and urine) samples.

Our presentation will provide a brief overview of PaCT activities in the four African countries involved. It will then focus on practical challenges encountered in the pilot studies and how they were addressed, with special reference to the South African pilot study. These challenges include funding procurement, standardisation of methods across countries, obtaining approval for the study, participant recruitment, data collection and analysis. 